\title{
The internal jugular vein as an alternative venous access for a revision of a fractured implantable cardioverter-defibrillator lead
}

Jong Yop Pae ${ }^{1}$, Yoon-Nyun Kim ${ }^{1}$, Min Young Do ${ }^{1}$, Hyoung-Seob Park ${ }^{1}$, Seongwook Han ${ }^{1}$, Seung-Ho Hur ${ }^{1}$, and Sae-Young Choi ${ }^{2}$

${ }^{1}$ Division of Cardiology, Department of Internal Medicine, ${ }^{2}$ Department of Thoracic and Cardiovascular Surgery, Keimyung University Dongsan Medical Center, Daegu, Korea

Received: July 13, 2015

Revised : August 13, 2015

Accepted: August 18, 2015

Correspondence to

Yoon-Nyun Kim, M.D.

Division of Cardiology,

Department of Internal Medicine,

Keimyung University Dongsan

Medical Center, 56 Dalseong-ro,

Jung-gu, Daegu 41931, Korea

Tel: +82-53-250-7432

Fax: +82-53-250-7034

E-mail:ynkim@dsmc.or.kr
To the Editor,

The Sprint Fidelis Model 6949 lead is a transvenous high-voltage implantable cardioverter-defibrillator (ICD) lead made by Medtronic Inc. (Minneapolis, MN, USA). Medtronic issued a recall of its Fidelis lead in October 2007 because a $15 \%$ to $20 \%$ rate of lead-related complications was noted after almost 5 years of follow-up [1]. The most common cause of lead failure was a fracture of the pacing-sensing wire. When the lead fracture occurs, the ICD fails to deliver needed shocks or delivers repeated inappropriate shocks. Therefore, patients who have Fidelis leads often experience an additional lead insertion or replacement.

Previous studies reported that after pacemaker or ICD implantations, a total or partial obstruction of the access vein rarely causes immediate clinical problems. However, venous obstructions occur relatively frequently and if the need for a lead revision or additional lead insertion arises, a different access route is required. Alternative methods have been studied, such as the utilization of other vessels or surgery $[2,3]$. We report a case in which the patient had both subclavian vein obstruction and underwent an additional pacing-sensing lead insertion through the internal jugular vein (IJV) for a fractured Fidelis lead.

An 81-year-old woman presented with an abnormal beeping sound from her ICD. She had a history of a coronary bypass surgery in 2002. The ICD (Medtronic MAXIMO DR, Sprint Fidelis Model 6949) was implanted in 2006 due to sustained ventricular tachycardia with loss of consciousness. She was found to have a complete occlusion of the left subclavian vein and the right subclavian vein was chosen for the ICD implantation. The ICD generator was replaced 4 years ago using the same Fidelis lead because the relatively high risk of the extraction of the lead and her old age were considered. She had been doing well without any events while the ICD was implanted. The patient had heard a beeping sound from the ICD. A device interrogation found a high impedance of the Fidelis lead (2,660 ohms) and many short V-V intervals with inappropriate shocks. We decided to add an additional pacing-sensing lead but a right subclavian vein occlusion with collateral flow to the superior vena cava was found on venography (Fig. 1). The right IJV was selected as an alternative vascular access. Following asepsis of the lateral cervical region and local anesthesia, the IJV was punctured without any difficulty between the sternal 


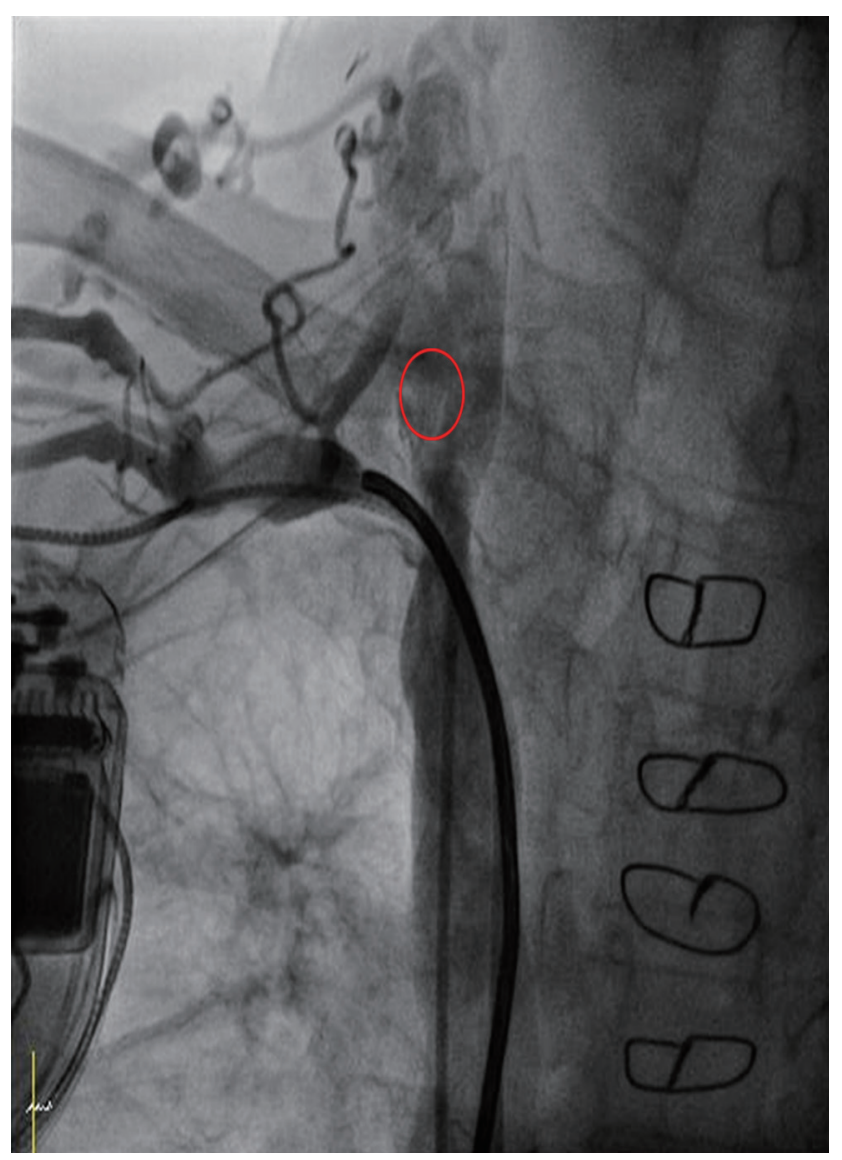

Figure 1. Venogram showing the implanted lead and tight stenosis of the right subclavian vein. Right internal jugular vein as an alternative vascular access (circle).

and clavicular belly of the sternocleidomastoid muscle (Fig. 2). An additional Sprint Qurattro Secure 6935 lead (Medtronic Inc.) was implanted in the right ventricle through a 9-Fr Peel-Away sheath. The lead was secured at the entry site. A subcutaneous tunnel was made from the entry site to the generator pocket through the anterior aspect of the clavicle. The additional lead was placed in the pocket through the subcutaneous tunnel (Fig. 3). The additional lead replaced the pacing-sensing part of the Fidelis lead and was connected to the pre-existing generator. The incision was closed layer by layer. The patient was discharged 7 days after the procedure without any complications.

The Fidelis lead is a 6.6-Fr bipolar high-voltage lead that had become popular because of its ease of handling. However, the Fidelis lead began to fracture soon after it was introduced in September 2004 and was withdrawn from the market in October 2007. The rate of Fidelis fail-

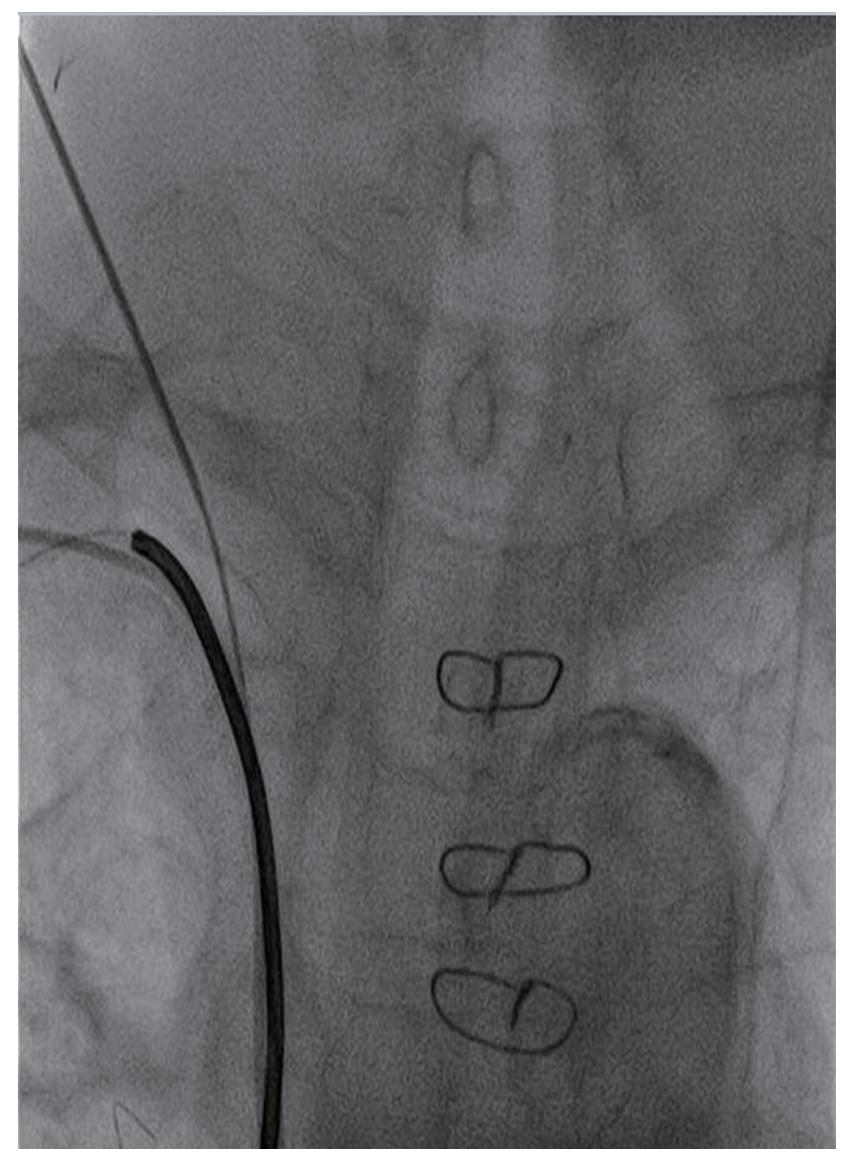

Figure 2. Chest radiograph showing the puncture of the right internal jugular vein.

ures continues to increase over time. Hence, as time goes on, the necessity for a lead revision increases.

Obstruction of the access vein is a well-known complication of ICD implantations. Obstructions are most commonly asymptomatic and symptomatic cases are known infrequently [4]. The presence of an obstruction in the access vein can make follow-up procedures, either for lead revisions or implantations of additional leads, difficult. Mostly, the technique used for the lead implantation was a subclavian puncture or cephalic vein cutdown. Several methods have been used to avoid surgery and general anesthesia, such as venoplasty, dilatation of a chronic total venous occlusion, or femoral access [2,3]. Pires et al. [5] reported that the right IJV can be used as a safe access for placement of permanent electrodes in cases where a left vein access cannot be used or when a right vein access is technically difficult.

In our case, we present a patient with a fractured Fidelis ICD lead and in which both subclavian veins were oc- 


\section{KJIM}

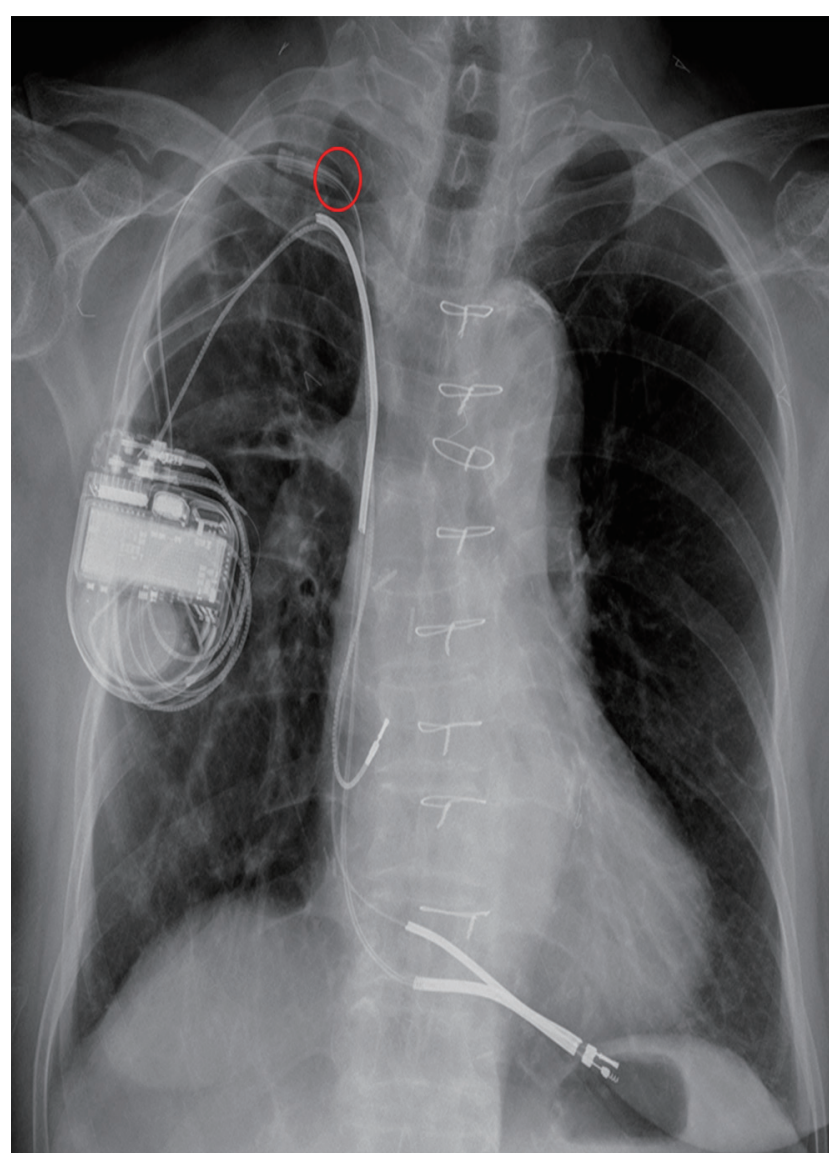

Figure 3. Chest radiograph showing the implanted lead configuration. Location of the puncture site of the right internal jugular vein (circle).

cluded. Due to inappropriate shocks owing to oversensing and high impedances, it was considered to be a lead fracture. Complications of lead revisions associated with extractions might be serious due to severe adhesions observed in venography and an additional lead implantation was planned. The left subclavian vein had previously been found to be occluded and in the venography, a right subclavian vein occlusion was also confirmed. We approached the right IJV as an alternative. We believed that the lead placement via the IJV with a subcutaneous tunnel to the subclavicular fossa could be an alternative method for the cardiac device implantation in cases with a subclavian vein occlusion.

Keywords: Jugular veins; Defibrillators; Lead

\section{Conflict of interest}

No potential conflict of interest relevant to this article was reported.

\section{Acknowledgments}

The research was supported by the Ministry of Science, ICT and Future Planning through the Development for IT.SW industrial convergence original technology (ID: Ro101-15-0147). We thank Mr. John Martin for his linguistic assistance.

\section{REFERENCES}

1. Birnie DH, Parkash R, Exner DV, et al. Clinical predictors of Fidelis lead failure: report from the Canadian Heart Rhythm Society Device Committee. Circulation 2012;125:1217-1225.

2. McCotter CJ, Angle JF, Prudente LA, et al. Placement of transvenous pacemaker and ICD leads across total chronic occlusions. Pacing Clin Electrophysiol 2005;28:921925 .

3. YousefZ, Paul V, Leyva F. Cardiac resynchronization via the femoral vein: a novel method in cases with contraindications to the pectoral approach. Europace 2006;8:144146.

4. Williams EH, Tyers GF, Shaffer CW. Symptomatic deep venous thrombosis of the arm associated with permanent transvenous pacing electrodes. Chest 1978;73:613615 .

5. Pires LA, Hassan SA, Johnson KM. Coronary sinus lead placement via the internal jugular vein in patients with advanced heart failure: a simplified percutaneous approach. J Interv Card Electrophysiol 2005;12:157-162. 